\title{
Determinación de la Tasa de Servicio y de los Factores que la Afectan en Establos de Lechería Intensiva de Lima, Perú
}

\author{
Determination of Service Rate and Factors that Affect It in Intensive \\ Dairy Farms of Lima, Peru \\ Rocío Silvia Sandoval Monzón ${ }^{1,3}$, Luis Felipe Ruiz García ${ }^{1}$, \\ Fernando Demetrio Carcelén Caceres ${ }^{2}$
}

\section{Resumen}

El presente trabajo tuvo como objetivo determinar la tasa de servicio (TS) de establos de lechería intensiva de Lima, Perú, e identificar los factores que la afectan. Se trabajó con 144 unidades experimentales, denominadas establo-mes. Cada una estuvo conformada por 60 vacas seleccionadas al azar, las cuales debían tener más de 60 días en lactación durante el mes de estudio y su fecha de concepción debía ser después de la fecha de inicio del mes de estudio. Se trabajó con los registros de cuatro establos de las provincias de Huaura, Lima y Cañete en el periodo entre agosto de 2010 y julio de 2013. Se evaluó la posible influencia sobre la TS de los días en lactación (DEL), nivel productivo (NPx), número de partos (NP), porcentaje de vacas con uso de prostaglandinas $(\% \mathrm{PG})$, porcentaje de vacas bajo un programa de inseminación artificial a tiempo fijo (\%IATF) y del índice temperatura-humedad máximo (ITH Máx). Asimismo, se evaluaron los factores establo, año y frecuencia de ordeño. La TS fue de $44.6 \%$. El \%IATF, ITH Max y NP fueron significativos $(\mathrm{p}<0.05)$, con coeficientes de regresión de $0.240,-0.655 \mathrm{y}$ -5.858 , respectivamente. Así mismo, la interacción establo-año y la variable establo fueron altamente significativas $(\mathrm{p}<0.001)$. Se concluye que la TS en los cuatro establos de Lima se encuentra por debajo del ideal y posee un gran potencial de mejora.

Palabras clave: tasa de servicio, establos lecheros, Lima, prostaglandina, inseminación a tiempo fijo

\footnotetext{
${ }^{1}$ Clínica de Animales Mayores, Facultad de Medicina Veterinaria, Universidad Nacional Mayor de San Marcos, Lima, Perú

${ }^{2}$ Laboratorio de Bioquímica, Nutrición y Alimentación Animal, Facultad de Medicina Veterinaria, Universidad Nacional Mayor de San Marcos, Lima, Perú

${ }^{3}$ E-mail: rocio.sandoval@unmsm.edu.pe
}

Recibido: 19 de agosto de 2016

Aceptado para publicación: 24 de noviembre de 2016 
This study aimed to determine the service rate (TS) in intensive dairy farms in Lima, Peru and to identify the factors affecting it. For this purpose, 144 experimental units (farm-month units) were used. Each unit consisted of 60 randomly selected cows who had more than 60 days in lactation during the study month and their conception date should be after the start date of the study month. Records of four dairy farms in the provinces of Huaura, Huaral, Lima and Cañete in the period of August 2010 to July 2013 were assessed. The effect of days in milk (DEL), production level (NPX), parity (NP), the percentage of inseminated cows by using prostaglandins (\%PG), percentage of inseminated cows under fixed-time artificial insemination (\%TAI) and maximum temperature-humidity index (THI Max) on service rate were evaluated. Moreover, farm, year, season and milking frequency were evaluated. TS was $44.6 \%$. The $\% \mathrm{TAI}, \% \mathrm{THI}$ and NP were highly significant ( $\mathrm{p}<0.05$ ), with a regression coefficient of $0.240,-0655$ and -5.858 respectively. Also, the interaction year $\mathrm{x}$ farm and farms were highly significant $(p<0.001)$. It is concluded that TS in the four dairy farms in Lima is below the ideal target and therefore, there is a great potential for improvement.

Key words: service rate, dairy farms, Lima, prostaglandin, fixed-time artificial insemination

\section{INTRODUCCIÓN}

El aspecto reproductivo del hato es una de las principales preocupaciones en toda ganadería lechera; sin embargo, su evaluación y monitoreo continúan siendo un desafío (Ferguson y Skidmore, 2013). La evaluación del comportamiento reproductivo de los establos lecheros ha ido cambiando con el tiempo. Los parámetros reproductivos clásicos como el intervalo entre partos, el número de días abiertos y el intervalo parto-concepción han ido perdiendo la importancia que tenían (Fetrow et al., 1990; Fetrow et al., 2007) para el manejo reproductivo del día a día. Los índices clásicos no detectan de manera oportuna las fallas del desempeño reproductivo, de allí que actualmente se recomienda su evaluación empleando la tasa de preñez (Niles et al., 2001), la cual es un índice que refleja de forma objetiva la rapidez con que se preñan las vacas en el establo (Ferguson y Galligan, 2000). Depende directamente de la tasa de servicio mensual (vacas vacías que se sirvieron en el mes) y la tasa de concepción mensual (vacas servidas que preñaron en el mes).
Es un parámetro que define en forma indirecta la rentabilidad de un establo lechero (Ferguson y Galligan, 2000; Niles et al., 2001).

Diversos estudios a nivel nacional demuestran que el desempeño reproductivo del ganado lechero en el Perú es deficiente (Ortiz et al., 2009; Ruiz y Sandoval, 2014), siendo evidente que la tasa de preñez tiene mucho margen para mejorar (Ruizy Sandoval, 2014). Por otro lado, la búsqueda de animales de alta productividad, a través del mejoramiento genético, ha devenido que las vacas tengan una menor tasa de fertilidad (Lucy, 2001), lo que limita el esfuerzo para mejorar la tasa de preñez (Ferguson y Galligan, 2000, Fetrow et al., 2007).

La tasa de servicio (TS) se define como el porcentaje de vacas servidas del total de vacas que se encuentran aptas para ser servidas durante un periodo de tiempo determinado (Fetrow et al., 2007; Murray, 2009). La TS evalúa la dinámica del establo para lograr un mayor número de numero de vacas inseminadas (Fetrow et al., 2007). 
La gran mayoría de los administradores y veterinarios de los establos lecheros de Lima conoce cuál es su tasa de concepción, pero muy pocos conocen cuál es su tasa de servicio. Centran sus esfuerzos en mejorar las tasas de concepción; sin embargo, la principal causa del incremento de días abiertos en los establos se debe a una diminución en la tasa de servicio (Bartolome y Archbald, 2011; Ferguson y Skidmore, 2013). Las bajas tasas de servicio no solo incrementan el intervalo de tiempo a la primera inseminación, sino que también incrementan el periodo de tiempo entre servicios (Stevenson y Call, 1983). Trabajos recientes sostienen que a pesar del incremento de la productividad lechera y de las bajas tasas de concepción, el incremento de la tasa de servicio en los establos permite obtener mejores tasas de preñez (LeBlanc, 2013).

Los factores que afectan la tasa de servicio han sido menos estudiados que aquellos que afectan la fertilidad (Cutullic et al., 2009). Entre los factores que influyen sobre la tasa de servicio se encuentran la pobre expresión del celo (Stevenson, 2001), fallas en la detección de celos, problemas de salud reproductiva periparto, tipo de suelo, movimiento de animales dentro del hato, número de ordeños, número de parto, nivel productivo y estrés calórico (Lopez et al., 2004; Cutullic et al., 2009). Por otro lado, se disponen de protocolos hormonales de sincronización de celo con inseminación a tiempo fijo y uso de prostaglandinas (PG) que reemplazan la detección de celo (Stevenson, 2001; Bartolome y Thatcher, 2011). Estos programas se pueden implementar al primer servicio, pero es complicado su uso en servicios posteriores, y pueden retrasar el intervalo entre inseminaciones (Bartolome y Archbald, 2011).

El presente trabajo tuvo la finalidad de identificar los factores asociados a la tasa de servicio de los establos de crianza intensiva de Lima.

\section{Materiales y Métodos}

El estudio fue de tipo observacional. Se evaluaron los registros de cuatro establos lecheros de crianza intensiva de las provincias de Huaura, Lima y Cañete, en Lima, Perú. El periodo evaluado fue de tres años, el cual se inició en agosto de 2010 y finalizó en julio de 2013.

\section{Establos y Animales}

La unidad de estudio del presente trabajo es la «unidad establo-mes», la cual está conformada por un conjunto de 60 vacas dentro de cada mes y de cada establo. Este conjunto de vacas fue seleccionado al azar dentro de cada grupo de vacas elegibles.

Los establos tenían un sistema de producción lechera intensiva, empleaban una alimentación a base a forraje y concentrado, contaban con ordeño mecánico, utilizaban inseminación artificial y tenían más de 200 vacas en producción.

Las vacas para ser elegidas, debían encontrarse en el registro de control de ordeño del mes de estudio, tener más de 60 días en lactación durante el mes de estudio y su fecha de concepción debía ser después de la fecha de inicio del mes de estudio. Fueron excluidas del estudio las vacas que presentaron algún problema sanitario grave o que fueron eliminadas del establo dentro del mes de estudio.

El periodo de estudio de cada vaca era hasta que la vaca preñe o termine el mes de estudio. Se trabajó con un total de 144 unidades establo-mes ( 3 años x 4 establos x 12 meses), recolectados de un total de 8640 registros.

\section{Variables Reproductivas y Productivas}

Para calcular los parámetros productivos y reproductivos de cada unidad establo- 
mes se recolectaron los datos de los registros de los establos. Asimismo, se recolectaron los datos meteorológicos del Servicio Nacional de Meteorología e Hidrología del Perú (SENAMHI) de las estaciones más cercanas a los establos de estudio para el cálculo de índice temperatura-humedad máximo.

El cálculo de los parámetros evaluados se describe a continuación:

Tasa de servicio establo-mes. La tasa de servicio (TS) establo-mes fue calculada según la fórmula de Fetrow et al. (1990):

$$
\mathrm{TS}=\frac{\mathrm{N}}{\left(\frac{\mathrm{D}}{21}\right)} \times 100
$$

donde $\mathrm{N}$ es la sumatoria de todos los servicios realizados en las vacas elegidas de cada establo en cada mes de estudio, y D es la sumatoria de días del ciclo estral (DCE) de todas las vacas elegidas de cada establo en cada mes de estudio.

En el caso de las vacas que no preñaron y participaron durante todo el mes de estudio, el DCE fue igual a los días del mes, y en el caso de las vacas que preñaron dentro del mes de estudio, los días de ciclo estral fueron la cantidad de días que participaron en el mes. En caso de haberse utilizado PG para reducir el intervalo del ciclo estral, se agregaron siete días al ciclo estral por cada dosis de PG empleada en el mes de estudio (Fetrow et al., 1990).

Días en lactación establo-mes (DEL). Se refiere al promedio aritmético de los días en lactación de las vacas que forman parte de cada unidad de estudio (Fetrow et al., 1990).

Nivel productivo establo-mes (NPx). Se refiere al promedio aritmético del nivel productivo de las vacas en cada establo-mes. Se calculó mediante la sumatoria de la producción de leche desde el día uno hasta el día 305 de lactación, estimada mediante la curva de lactación. Se utilizó la fórmula gamma in- completa lineal propuesta por Singh y Gopal (1982) para el cálculo de la curva de lactación. Esta fórmula fue modificada para obtener la producción de leche corregida a dos ordeños, sesenta meses de edad, cuatro partos y se eliminó el efecto del mes de parto. Estas correcciones fueron estimadas mediante la inclusión de términos lineales a la ecuación. Los parámetros de cada curva de lactación fueron estimados empleando cinco controles de ordeño para cada vaca en los días 30, 60, 90, 120 y 240 de la lactación.

Índice temperatura-humedad máximo establo-mes (ITH max). Se refiere al promedio aritmético del ITH máx de cada día de cada zona de cada mes de estudio. Para esto, se utilizó la fórmula presentada por Frank Wiersma (Bouraoui et al., 2002). ITH $=0.81$ $\mathrm{x} \mathrm{T}^{\circ} \max +\mathrm{HRmin} / 100 \mathrm{x}\left(\mathrm{T}^{\circ}-14.4\right)+46.4$, donde $\mathrm{T}^{\circ}$ max es la temperatura máxima registrada en el día y HR min es la humedad relativa mínima registrada en el día.

Promedio de número de partos establo-mes (NP). Se refiere al promedio aritmético del número de partos de las vacas que forman parte de cada unidad de estudio.

Porcentaje de vacas con uso de prostaglandinas $(\% \mathrm{PG})$. Se refiere a la relación porcentual entre el número de dosis de prostaglandinas empleadas y el número de vacas que forman parte de cada unidad de estudio.

Porcentaje de vacas bajo un programa de inseminación artificial a tiempo fijo (\% IATF). Se refiere a la relación porcentual entre el número de vacas que fueron sometidas a IATF (Ovsynch, CIDR, otros) y aquellas que forman parte de cada unidad de estudio.

\section{Diseño Experimental}

Se determinó la TS global para los cuatro establos lecheros de crianza intensiva. Asimismo, se evaluó el efecto de los factores que influyen sobre la TS, mediante el siguiente modelo general lineal: 
$\mathrm{Y}_{\mathrm{ijk}}=\mu+\mathrm{b} 1 \mathrm{xX}_{1}+\mathrm{b} 2 \mathrm{xX}_{2}+\mathrm{b} 3 \mathrm{xX}_{3}+\mathrm{b} 4 \mathrm{xX}_{4}+$ $\mathrm{b} 5 \mathrm{xX}_{5}+\mathrm{b} 6 \mathrm{xX}_{6}+\mathrm{E}_{\mathrm{i}}+\mathrm{A}_{\mathrm{j}}+\mathrm{E}_{\mathrm{i}} \mathrm{xA}_{\mathrm{j}}+=\mathrm{O}_{\mathrm{k}}\left(\mathrm{E}_{\mathrm{i}}\right)+$ $\mathrm{e}_{\mathrm{ijk}}$

donde:

$\mathrm{Y}_{\mathrm{ijk}}$ es la tasa de servicio mensual de la observación ijkl-ésima $\mu$ es la media

$\mathrm{b}_{1}, \mathrm{~b}_{2}, \mathrm{~b}_{3}, \mathrm{~b}_{4}, \mathrm{~b}_{5}, \mathrm{~b}_{6}$ son los coeficientes de regresión

$\mathrm{X}_{1}$ es el ITH máximo promedio en el establo i-ésimo y del mes de estudio j-ésimo

$\mathrm{X}_{2}$ son los DEL promedio en el establo i-ésimo $\mathrm{y}$ del mes de estudio j-ésimo

$\mathrm{X}_{3}$ es el promedio del número de partos (NP) en el establo i-ésimo y del mes de estudio jésimo

$\mathrm{X}_{4}$ es el \%PG en el establo i-ésimo y del mes de estudio j-ésimo

$\mathrm{X}_{5}$ es el \%IATF en el establo i-ésimo y del mes de estudio j-ésimo

$\mathrm{X}_{6}$ es el NPx promedio en el establo i-ésimo y del mes de estudio j-ésimo

$\mathrm{E}_{\mathrm{i}}$ es el efecto del establo i-ésimo $(\mathrm{i}=1,2,3,4)$

$A_{j}$ es el efecto del año de estudio j-ésimo $(j=1,2,3)$

$E_{i} \times A_{j}$ es la interacción entre el establo iésimo y el año de estudio j-ésimo

$\mathrm{O}_{k}\left(E_{\mathrm{i}}\right)$ es el efecto de la frecuencia de ordeño $\mathrm{k}$-ésima dentro del establo i-ésimo $(\mathrm{k}=2,3)$

$\mathrm{e}_{\mathrm{ijk}} \mathrm{k}$ es el error aleatorio de la observación ijkésima

\section{Análisis Estadístico}

Para la determinación de la TS global, los datos fueron promediados para obtener los valores medios; asimismo, se determinó el intervalo de confianza al 95\%. Para determinar los factores que influyeron la TS se empleó el análisis de varianza, para evaluar la normalidad de los residuos del modelo se empleó la prueba de Shapiro-Wilk y para evaluar la homogenidad de varianza se empleó la prueba de Levene.

Se estimaron los coeficientes de regresión de las covariables evaluadas y se realizó una prueba de «t» de Student para determinar los niveles de significancia al 5\%. Las medias marginales fueron estimadas para las variables que fueron significativas. Las covariables fueron corregidas por las medias y se compararon los efectos principales. Para esto se realizó una comparación por pares utilizando el ajuste de diferencia menor significativa.

Los datos fueron analizados mediante el paquete estadístico SPSS v. 22.0 para Windows.

\section{Resultados}

La TS en los establos lecheros de crianza intensiva de Lima fue de $44.6 \pm 2.0 \%$, con un rango de variación de 15.4 a $72.6 \%$. En la Figura 1 se presenta la tasa de servicio mensual en los cuatro establos. Se puede observar que el mayor porcentaje $(34.0 \%)$ de las tasas de servicio fue menor a $40 \%$, mientras solo el $11.8 \%$ superó la tasa de servicio de $60 \%$.

En el Cuadro 1 se presentan los resultados del análisis de varianza para el modelo del estudio. La interacción entre las variables establo y año y la variable establo fueron altamente significativas $(\mathrm{p}<0.001)$, mas no así la variable año. Asimismo, la variable frecuencia de ordeño dentro del establo fue altamente significativa $(\mathrm{p}<0.01)$.

Al evaluar las seis covariables del estudio, se encontró que \%IATF, ITH Máx y NP fueron estadísticamente significativas $(\mathrm{p}<0.05)$. Con respecto a la frecuencia de ordeño dentro de establo, solo un establo presentó grupos de vacas con dos frecuencias de ordeño, encontrándose que las vacas con tres ordeños presentaban una TS mayor que aquellas con dos ordeños (49.9 vs. $43.2 \%$, $\mathrm{p}<0.01$ ). Esto puede deberse a que las vacas que se ordeñan tres veces son más observadas por el personal del establo, de allí que se puedan detectar una mayor cantidad de celos y, por lo tanto, una mayor TS. 


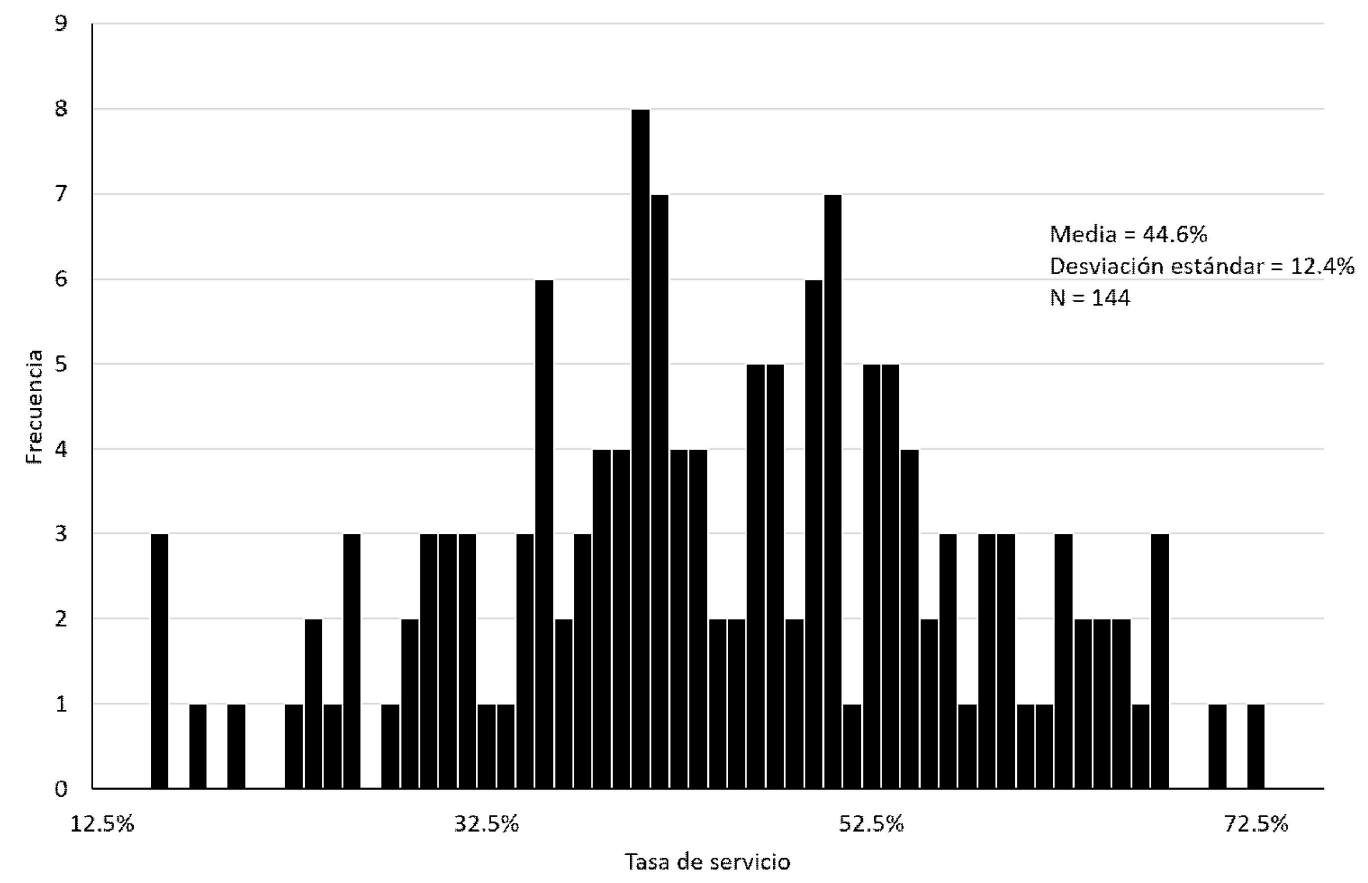

Figura 1. Histograma de frecuencia de la tasa de servicio en las unidades establo-mes $(n=144)$ en cuatro establos lecheros de crianza intensiva (Lima, Perú)

Cuadro 1. Resultados del análisis de varianza. Efecto de los factores que influyen sobre la tasa de servicio en cuatro establos lecheros de crianza intensiva (Lima, Perú)

\begin{tabular}{lcccc}
\hline Fuente $^{1}$ & $\begin{array}{c}\text { Grados de } \\
\text { libertad }\end{array}$ & $\begin{array}{c}\text { Cuadrado } \\
\text { medio }\end{array}$ & F & Sig. \\
\hline Establo & 3 & 2323.696 & 22.041 & 0.000 \\
Año & 2 & 274.162 & 2.600 & 0.077 \\
Establo * Año & 6 & 578.185 & 5.484 & 0.000 \\
Frecuencia de ordeño & 1 & 748.560 & 7.100 & 0.008 \\
DEL & 1 & 135.382 & 1.284 & 0.259 \\
NPx & 1 & 29.958 & 0.284 & 0.595 \\
NP & 1 & 601.871 & 5.709 & 0.018 \\
\%PG & 1 & 53.959 & 0.512 & 0.475 \\
\%IATF & 1 & 616.383 & 5.846 & 0.017 \\
ITH Máx & 1 & 657.623 & 6.238 & 0.014 \\
Error & 160 & 105.428 & & \\
\hline Total & 178 & & & \\
\hline
\end{tabular}

${ }^{1}$ DEL: días en lactación establo-mes; NPx: nivel productivo establo-mes; NP: promedio de número de partos establo-mes; \%PG: porcentaje de vacas que emplearon prostaglandinas; \%IATF: porcentaje de vacas bajo un programa de inseminación artificial a tiempo fijo; ITH Máx: índice temperatura-humedad máximo establo-mes 
Cuadro 2. Coeficientes de regresión, error estándar de la media y nivel de significancia para NP, \%IATF e ITH-Max en cuatro establos lecheros de crianza intensiva (Lima, Perú)

\begin{tabular}{lcccccc}
\hline & & & & & \multicolumn{2}{c}{$\begin{array}{c}\text { Intervalo de confianza } \\
\text { al 95\% }\end{array}$} \\
\cline { 6 - 7 } & & $\begin{array}{c}\text { Error } \\
\text { estándar }\end{array}$ & $\mathrm{t}$ & Sig. & $\begin{array}{c}\text { Límite } \\
\text { inferior }\end{array}$ & $\begin{array}{c}\text { Límite } \\
\text { superior }\end{array}$ \\
\hline DEL & 0.039 & 0.035 & 1.133 & 0.259 & -0.029 & 0.107 \\
NPx & 0.001 & 0.002 & 0.533 & 0.595 & -0.003 & 0.006 \\
NP & -5.858 & 2.452 & -2.389 & 0.018 & -10.699 & -1.016 \\
\%PG & -0.052 & 0.073 & -0.715 & 0.475 & -0.196 & 0.092 \\
\%IATF & 0.240 & 0.099 & 2.418 & 0.017 & 0.044 & 0.436 \\
ITH Max & -0.655 & 0.262 & -2.498 & 0.014 & -1.173 & -0.137 \\
\hline
\end{tabular}

${ }^{1}$ DEL: días en lactación establo-mes; NPx: nivel productivo establo-mes; NP: promedio de número de partos establo-mes; \%PG: porcentaje de vacas que emplearon prostaglandinas; \%IATF: porcentaje de vacas bajo un programa de inseminación artificial a tiempo fijo; ITH Máx: índice temperatura-humedad máximo establo-mes

En el Cuadro 2 se presentan los coeficientes de regresión de las covariables en estudio. El coeficiente de regresión de 0.24 para \%IATF $(\mathrm{p}<0.05)$ indica que con el incremento de una unidad en el \%IATF en el establo se puede incrementar en $0.24 \%$ la tasa de servicio (Figura 2). En forma similar, el coeficiente de regresión de -5.8 para NP $(p<0.05)$ indica que el incremento del promedio del número de partos en una unidad disminuye la tasa de servicios en $5.8 \%$ (Figura 3 ), lo cual podría indicar, además, que la edad está relacionada inversamente con la tasa de servicios. Asimismo, el incremento de una unidad del ITH Máx disminuye la tasa de servicio en $0.7 \%(p<0.05)$ (Figura 4). Las demás covariables del estudio no fueron significativas, no encontrándose efecto del \% $\mathrm{PG}$, NPx, ni de los DEL.

En la Figura 5 se representan los promedios de la tasa de servicio de los cuatro establos bajo evaluación, corregidos mediante los valores promedio de las variables involucradas en el estudio. La TS del establo
$1(59.3 \%)$ fue significativamente superior que los establos 2,3 y $4(41.9,44.2$ y $33.1 \%$ respectivamente), no habiendo diferencia estadística entre los establos 2 y 3 , ni entre los establos 2 y 4 .

\section{Discusión}

Para lograr una óptima eficiencia reproductiva en un establo lechero se recomienda alcanzar un intervalo entre partos de 13 meses y para lograrlo se requiere conseguir una tasa de preñez de 28\% (Bartolome y Archald, 2011). Es evidente que la tasa de preñez (porcentaje de vacas preñadas del total de vacas disponibles en un periodo de 21 días) en los establos lecheros de Lima tiene mucho margen por mejorar, ya que en un estudio previo se observó solo un 10.1\% (Ruiz y Sandoval, 2014). Esta situación es complicada dado que la presión de selección para la obtención de animales de alta producción lechera mediante el mejoramiento genético a través de pruebas de progenie está 


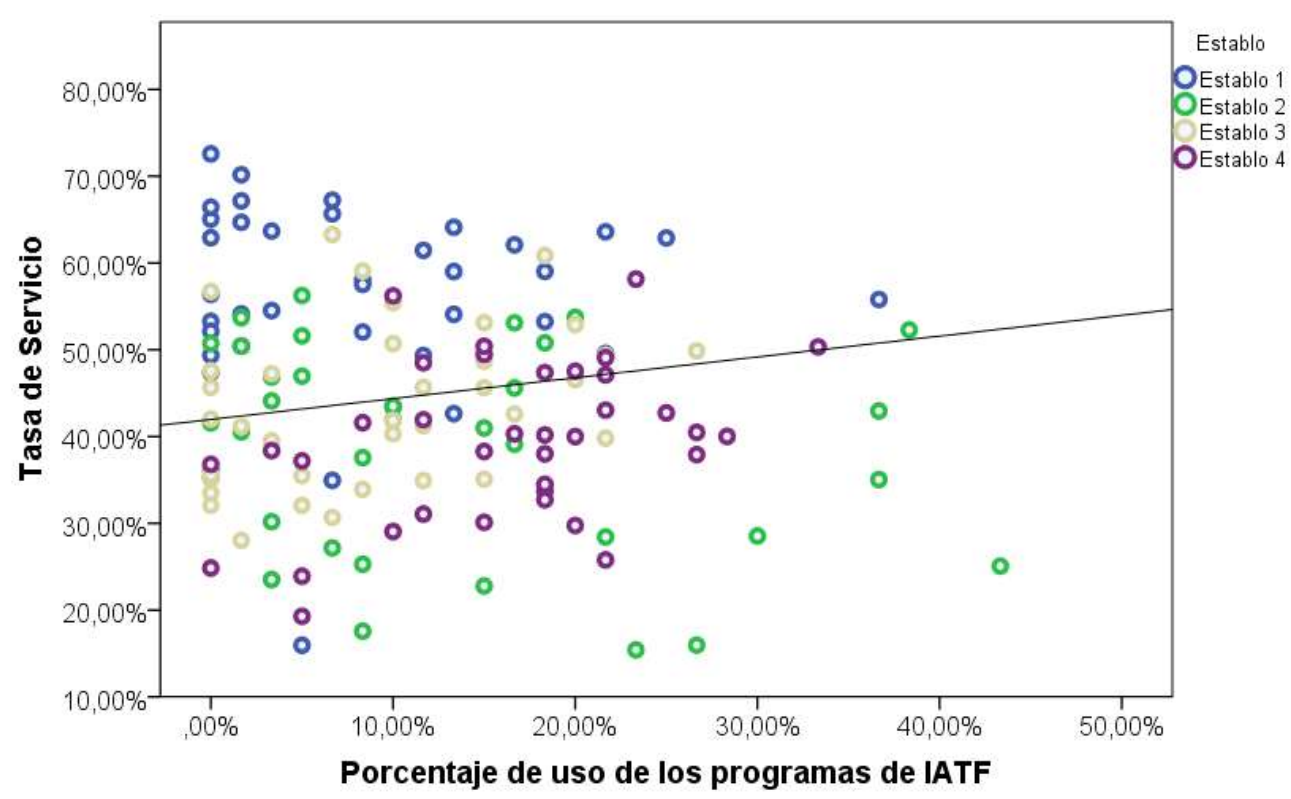

Figura 2. Dispersión de puntos entre la tasa de servicio mensual (TS) y el porcentaje de uso de los programas de inseminación artificial a tiempo fijo (IATF) según establo y la curva estimada de la TS según el IATF $(\mathrm{TS}=41.9766+0.240 *$ IATF $)$

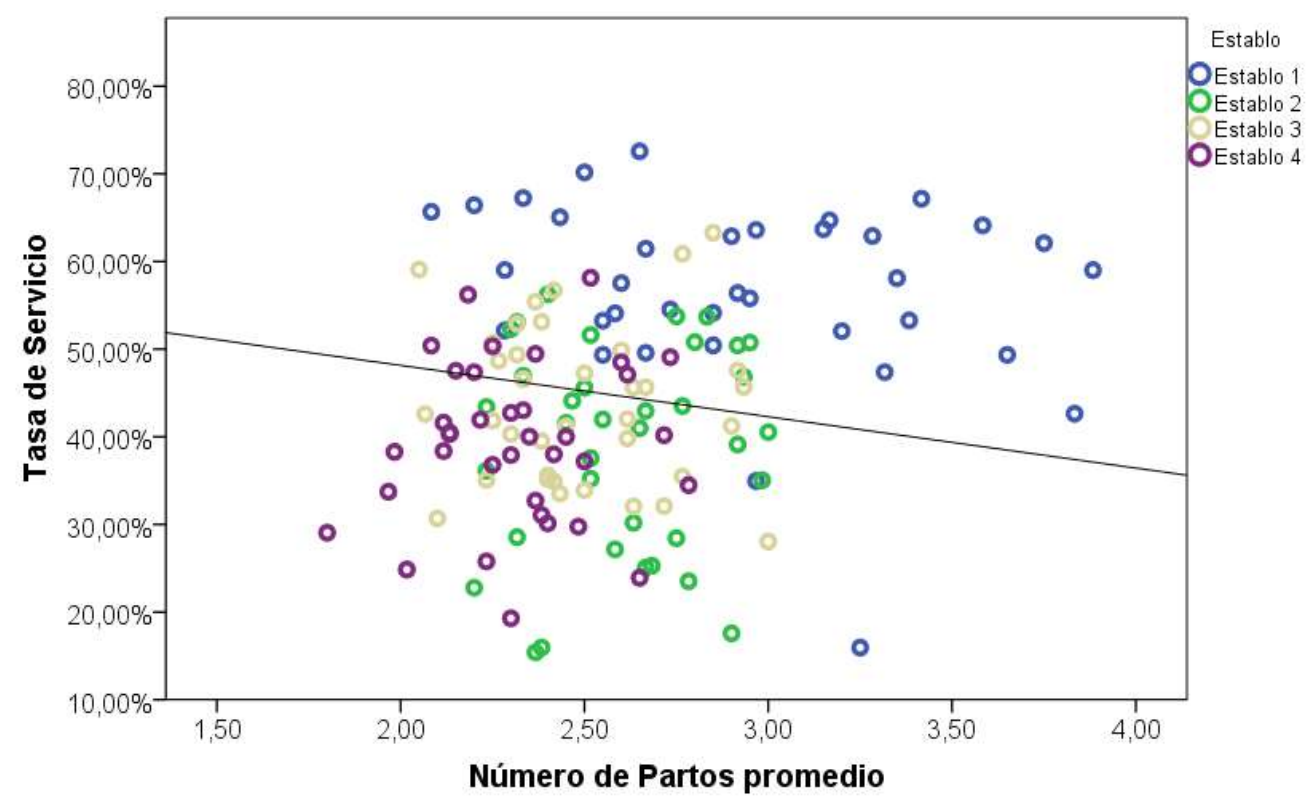

Figura 3. Dispersión de puntos entre la tasa de servicio mensual (TS) y el promedio del número de partos (NP) según establo y la curva estimada de la TS y el NP $(\mathrm{TS}=59.86322$ $5.858 * \mathrm{NP})$ 


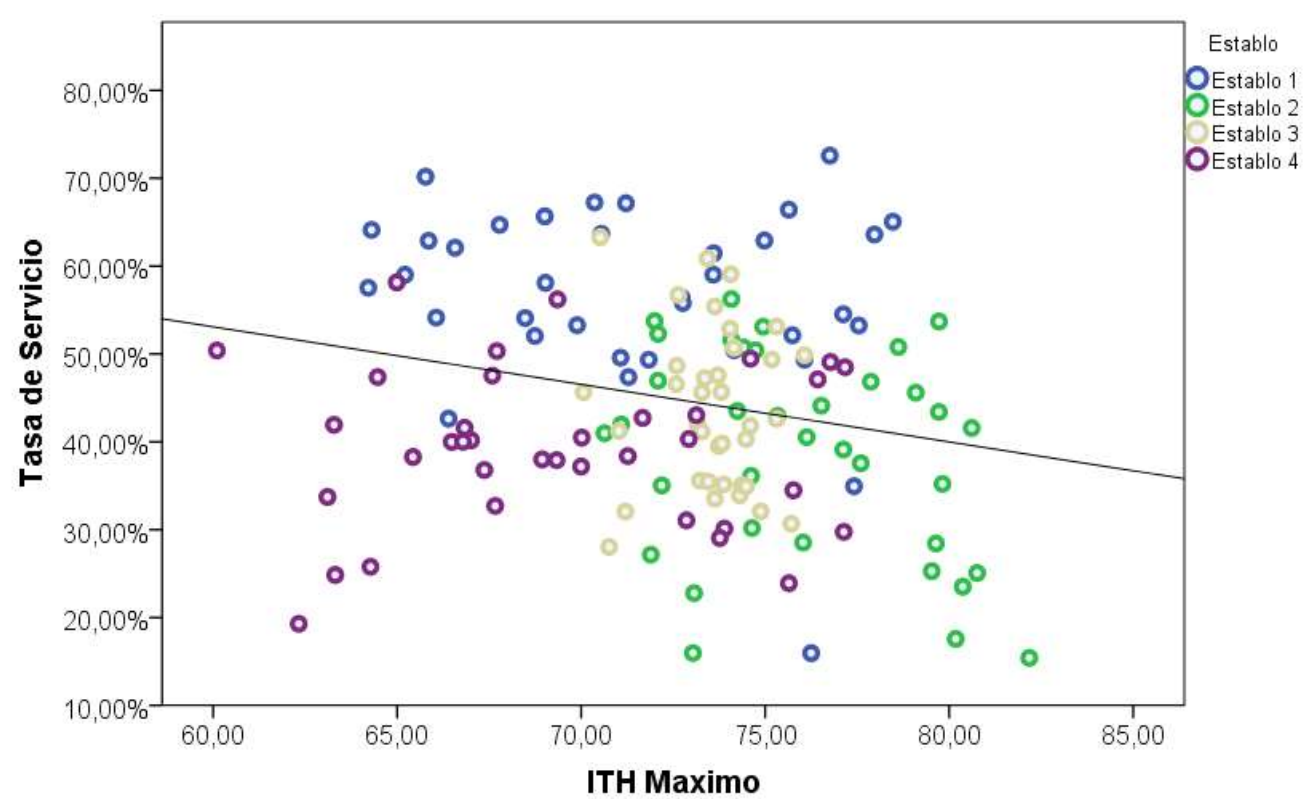

Figura 4. Dispersión de puntos entre la tasa de servicio mensual (TS) y el índice de temperatura humedad máximo (ITH Máx) según establo y la curva estimada de la TS y el ITH Máx $(\mathrm{TS}=92.39465-0.655 *$ ITH $)$

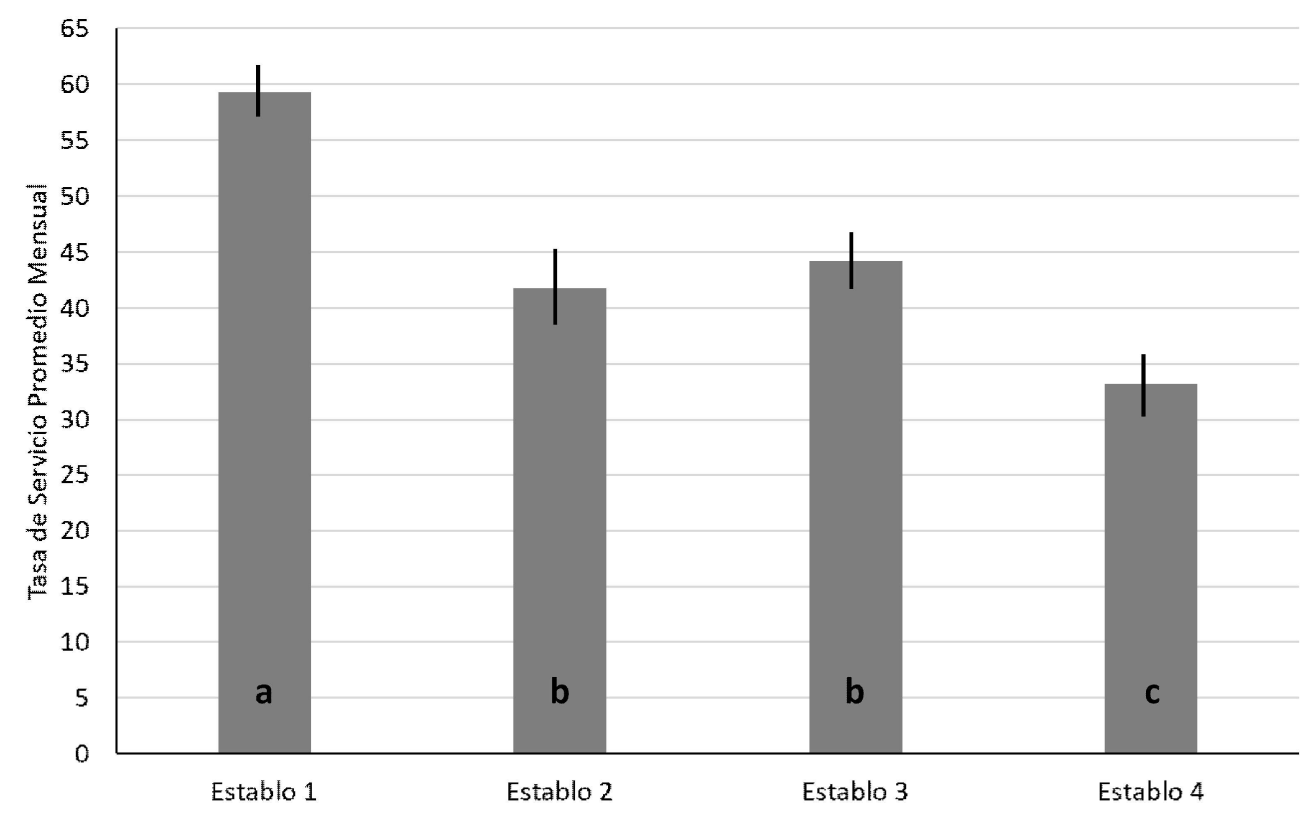

Figura 5. Medias estimadas y errores estándares de la media para la tasa de servicio mensual de cuatro establos lecheros de Lima, Perú. Las medias fueron calculadas empleando los promedios de los días en lactación (156 d), nivel productivo (9981 kg por campaña), número de partos (2.59), porcentaje de vacas con uso de PG (14.6\%), porcentaje de vacas bajo IATF (11.3\%) e índice de temperatura-humedad máximo (72.83). Letras diferentes al pie de las columnas indican diferencia estadística entre establos $(p<0.05)$ 
correlacionada con una baja tasa de concepción (Lucy, 2001; Olynk y Wolf, 2008).

La mayoría de los establos de crianza intensiva en Lima cuenta con un profesional encargado residente en el establo y con un asesor externo, lo cual significaría que se realiza una adecuada monitorización del desempeño reproductivo. Sin embargo, solo uno de los cuatro establos conocía el concepto de TS y realizaba el monitoreo de la TS dentro de sus parámetros reproductivos. Los resultados del presente trabajo determinaron que la TS en estos establos era de $45 \%$, lo que significa que solo 4.5 de cada 10 vacas elegibles son servidas cada 21 días en estos establos. Este valor está muy por debajo del valor recomendado de 70\% de TS (Bartolome y Archald, 2011; Fetrow et al., 2007).

Otro problema que pudo ser observado es que la TS solo superó el $60 \%$ en el $12 \%$ de los meses estudiados, lo que significa que la mayor parte del tiempo, los establos están dejando de servir a más del $50 \%$ de las vacas. Si a esto se suma la tasa de concepción de 30 a $40 \%$ en la zona (Ruiz y Sandoval, 2014), se tiene una tasa de preñez en los establos de Lima de $15 \%(0.44 * 0.35=0.15)$, muy por debajo del ideal.

Diversos autores sostienen que es más fácil mejorar la TS que la tasa de concepción en un establo lechero (Pecsok et al., 1994; Fricke, 2001). Esto se debe a que la TS tiene tres veces mayor variabilidad que la tasa de concepción (Pecsok et al., 1994). Sin embargo, un error muy común en la administración de un establo lechero es enfocar la atención únicamente en tratar de mejorar la tasa de concepción (aplicación de hormonas como la hormona liberadora de gonadotropinas o la hormona luteinizante al momento de la inseminación) y dejar de lado las técnicas y tecnologías que mejoran la TS (observación directa, uso de podómetros, empleo de parches detectores de celo, entre otros) (Fricke, 2001).
En una encuesta realizada en Estados Unidos, se concluyó que uno de los principales problemas reproductivos en ganado lechero eran las bajas TS (Caraviello et al., 2006), así como las bajas tasas de concepción y la presentación de infecciones uterinas (Caraviello et al., 2006, Fetrow et al., 2007, LeBlanc, 2013). Entre los principales problemas para mejorar la TS de un establo lechero de explotación intensiva se tiene a la baja expresión del celo y a los problemas administrativos del personal del establo para la detección visual del celo (Bartolome y Thatcher, 2011). La TS puede ser afectada por la calidad del celo, condiciones anovulatorias, gestión de la detección del celo y el uso de protocolos de inseminación a tiempo fijo (Stevenson, 2001).

En este estudio se encontró que el \%IATF influye significativamente la TS, concordando con los resultados de Stevenson (2001). Sin embargo, se debe precisar que el empleo de hormonas en establos lecheros de EUU involucra el 86\% de los primeros servicios y el $77 \%$ de los demás servicios (Caraviello et al., 2006), a diferencia de los establos de Lima donde el empleo de hormonas es bastante limitado. Por otro lado, el incremento en una unidad en el \%IATF en el establo incrementa la TS en $0.24 \%$, no siendo tan beneficioso como se esperaba, debido posiblemente a problemas logísticos, dado que se debe saber el estado reproductivo de los animales (solo se pueden sincronizar animales vacíos) y se deben planificar los protocolos de trabajo, los cuales pueden llevar tiempo y, por lo tanto, retrasar el intervalo entre inseminaciones (Bartolome y Archbald, 2011).

El empleo de la IATF puede ser una opción para realizar el primer servicio, pero para realizar los posteriores servicios, es necesario combinarlo con un eficiente programa de detección de celos, estrategias para la detección temprana de vacas no preñadas o el empleo de programas de resincronización 
(Bartolome y Archbald, 2011). Se debe considerar que las bajas TS no solo incrementan el intervalo de tiempo al primer servicio, sino que también incrementan el intervalo interservicios (Stevenson y Call, 1983). Por otro lado, el empleo de la IATF puede tener un efecto de desmotivación sobre el personal del establo para la correcta detección visual de los celos. El personal del establo debe comprender que los programas de IATF son un complemento y no un reemplazo de las labores de detección de celo (Bartolome y Archald, 2011).

Según los resultados del presente estudio, las vacas con mayor número de campañas tienen una menor probabilidad de ser servidas a pesar de encontrarse disponibles. Esto podría deberse a la producción de leche del animal, ya que los niveles de producción aumentan conforme se incrementa el número de partos. Según Lopez et al. (2004), las vacas primíparas producen menos leche que las multíparas y las vacas que presentan mayor producción de leche presentan un celo más corto que las vacas que producen menor cantidad de leche (6.9 y 10.6 horas, respectivamente).

Por otro lado, también se debe considerar que el incremento de una unidad en el ITH disminuye la TS en $0.7 \%$. Esto indica que conforme más alto es el ITH Máx, las vacas tienen una menor probabilidad de ser servidas a pesar de encontrarse disponibles. Esto concuerda con el estrés por calor (ITH Max>70), factor que reduce la eficiencia reproductiva del ganado lechero (Wolfenson et al., 2000). El estrés por calor altera la intensidad y la duración del estro y disminuye la actividad de monta (Nebel et al., 1997), lo cual reduce la probabilidad de detectar los celos.

El uso de la PG como método de manipulación hormonal es más común que los programas de IATF en los establos de Lima, debido a su menor costo. Sin embargo, se encontró que su empleo no afectó significativamente la TS. Esto podría deber- se a que, si bien su uso resulta en un acortamiento del ciclo estral y la presentación de celo, si no es detectado en el momento oportuno, se convierte en un nuevo celo perdido y no servido.

Las variables interacción establo por año y establo fueron altamente significativas, lo cual se debe a características de manejo propias de cada establo (personal, instalaciones, administración, ubicación, entre otros). La interacción entre el establo y año indica, además, una gran variabilidad de la TS en los establos en el transcurso del tiempo, lo cual podría ser explicada por cambios en el manejo, así como aumento en el número de animales y dificultades administrativas (LeBlanc, 2013).

En relación a las diferencias encontradas entre establos, se puede indicar que el establo 1, el cual tuvo la mejor TS, es el único que evalúa periódicamente la TS y posee instalaciones que le permiten una mejor visualización de los animales en celo; sin embargo, no brinda un incentivo económico a sus trabajadores para la detección de celo, lo cual podría ayudar a mejorar su TS (Bartolome y Archbald, 2011). Los establos 2 y 3 proporcionan un incentivo económico a los operarios que detectan celo (Olynk y Wolf, 2008), el establo 2 cuenta con un pasadizo central entre corrales que facilita la detección de celo, mientras que el establo 4 tiene una gran desventaja con sus instalaciones (Garbarino et al., 2004). El éxito en la detección de celo está directamente relacionado con la responsabilidad y capacidad de observación del personal encargado de la detección (Diskin y Screenam, 2000).

\section{Conclusiones}

- La tasa de servicio de establos lecheros de crianza intensiva del departamento de Lima es de $44.6 \%$, con un rango de variación bastante amplio, lo que representa un gran potencial de mejora. 
- El empleo de programas de inseminación artificial a tiempo fijo es un factor que influye positivamente sobre la tasa de servicio de los establos, aunque en forma limitada.

- $\quad$ El incremento de una unidad en el índice de temperatura-humedad máximo disminuye la tasa de servicios en $0.66 \%$.

\section{Literatura Cittada}

1. Bartolome JA, Archbald LF. 2011. Reproductive management in dairy cows. In: Risco CA (ed). Dairy production medicine. Iowa: John Wiley \& Sons. p 73-79.

2. Bartolome JA, Thatcher WW. 2011. Resynchronization of estrus, ovulation, and timed insemination. In: Risco CA (ed). Dairy production medicine. Iowa: John Wiley \& Sons. p 117-122.

3. Bouraoui R, Lahmar M, Majdoub A, Djemali M, Belyea R. 2002. The relationship of temperature-humidity index with milk production of dairy cows in a Mediterranean climate. Anim Res 51: 479-491. doi: 10.1051/animres:2002036

4. Caraviello DZ, Weigel KA, Fricke PM, Wiltbank MC, Florent MJ, Cook NB, Nordlund KV, et al. 2006. Survey of management practices on reproductive performance of dairy cattle on large US commercial farms. J Dairy Sci 89: 47234735. doi: 10.3168/jds.S0022-0302(06)72522-X

5. Cutullic E, Delaby L, Causeur D, Michel G, Disenhaus C. 2009. Hierarchy of factors affecting behavioural signs used for oestrus detection of Holstein and Normande dairy cows in a seasonal calving system. Anim Reprod Sci 113: 22-37. doi: 10.1016/j.anireprosci.2008.07.001

6. Diskin MG, Sreenan J. 2000. Expression and detection of oestrus in cattle. Reprod Nutr Dev 40: 481-491.
7. Ferguson JD, Galligan DT. 2000. Assessment of reproductive efficiency in dairy herds. Comp Cont Educ Pract 22: S150-S159.

8. Ferguson JD, Skidmore A. 2013. Reproductive performance in a select sample of dairy herds. J Dairy Sci 96: 1269-1289. doi: 10.3168/jds.2012-5805

9. Fetrow J, McClary D, Harman R, Butcher K, Weaver L, Studer E, Ehrlich J, et al. 1990. Calculating selected reproductive indices: recommendations of the American Association of Bovine Practitioners. J Dairy Sci 73: 78-90. doi: 10.3168/ jds.S0022-0302(90)78649-3

10. Fetrow J, Stewart S, Eicker S, Rapnicki $P$. 2007. Reproductive health programs of dairy herds: analysis of records for assessment of reproductive performance. In: Youngquist RS, Threlfall WR (eds). Current therapy in large animal theriogenology. $2^{\text {nd }}$ ed. Missouri, USA: Saunders Elsevier. p 473-489.

11. Fricke PM. 2001. Entendiendo la clave para una reproducción exitosa. Novedades Lácteas. Reproducción y Selección Genética $\mathrm{N}^{\circ}$ 606. Instituto Babcock. Universidad de Wisconsin. [Internet]. Disponible en: http:// babcock.wisc.edu/sites/default/files/ documents/productdownload/ du_606.es_.pdf

12. Garbarino EJ, Hernandez JA, Shearer JK, Risco CA, Thatcher WW. 2004. Effect of lameness on ovarian activity in postpartum Holstein cows. J Dairy Sci 87: 4123-4131. doi: 10.3168/ jds.S0022-0302(04)73555-9

13. LeBlanc SJ. 2013. Is a high level of milk production compatible with good reproductive performance in dairy cows? Animal Frontiers 3(4): 84-91.

14. Lopez H, Satter LD, Wiltbank MC. 2004. Relationship between level of milk production and estrous behavior of lactating dairy cows. Anim Reprod Sci 81: 209-223. doi: 10.1016/j.anireprosci.2003.10.009 
15. Lucy MC. 2001. Reproductive loss in high-producing dairy cattle: where will it end? J Dairy Sci 84: 1277-1293. doi: 10.3168/jds.S0022-0302(01)70158-0

16. Murray R. 2009. Evaluación reproductiva mensual. [Internet]. Disponible en: http://www.produccionanimal.com.ar/informacion_tecnica/ inseminacion_artificial/150evaluacion.pdf

17. Nebel RL, Jobst SM, Dransfield MBG, Pandolfi SM, Bailey TL. 1997. Use of a radiofrequency data communication system, Heat Watch ${ }^{\circledR}$, to describe behavioral estrus in dairy cattle. J Dairy Sci 80: 151-179.

18. Niles D, Eicker S, Stewart S. 2001. Using pregnancy rate to monitor reproductive management. In: Proc $5^{\text {th }}$ Western Dairy Management Conference. Las Vegas, USA.

19. Olynk NJ, Wolf CA. 2008. Economic analysis of reproductive management strategies on US commercial dairy farms. J Dairy Sci 91: 4082-4091. doi: 10.3168/ jds.2007-0858

20. Ortiz D, Camacho J, Echevarría L. 2009. Parámetros reproductivos del ganado vacuno en la cuenca lechera de Lima. Rev Inv Vet Perú 20: 196-202. doi: 10.15381/rivep.v20i2.606
21. Pecsok SR, McGilliard ML, Nebel RL. 1994. Conception rates. 1. Derivation and estimates for effects of estrus detection on cow profitability. J Dairy Sci 77: 3008-3015. doi: 10.3168/jds.S00220302(94)77242-8

22. Ruiz L, Sandoval R. 2014. Relación entre los parámetros reproductivos convencionales y los parámetros de eficiencia reproductiva de los establos lecheros de Lima. Spermova 4(1): 58-60.

23. Singh RP, Gopal R. 1982. Lactation curve analysis of buffaloes maintained under village conditions. Indian J Anim Sci 52: 1157-1160.

24. Stevenson JS, Call EP. 1983. Influence of early estrus, ovulation, and insemination on fertility in postpartum Holstein cows. Theriogenology 19: 367-375. doi: 10.1016/0093-691X(83)90092-4

25. Stevenson JS. 2001. Reproductive management of dairy cows in high milkproducing herds. J Dairy Sci 84(Suppl): E128-E143. doi: 10.3168/jds.S00220302(01)70207-X

26. Wolfenson D, Roth Z, Meidan R. 2000. Impaired reproduction in heatstressed cattle: basic and applied aspects. Anim Reprod Sci 60-61: 535-547. doi: 10.1016/S0378-4320(00)00102-0 